\title{
Gust Factors: Meteorologically Stratified Climatology, Data Artifacts, and Utility in Forecasting Peak Gusts
}

\author{
AUSTIN R. HARRIS AND JONATHAN D. W. KAHL \\ Atmospheric Science Program, Department of Mathematical Science, \\ University of Wisconsin-Milwaukee, Milwaukee, Wisconsin
}

(Manuscript received 15 May 2017, in final form 4 September 2017)

\begin{abstract}
Gust factors in Milwaukee, Wisconsin, are investigated using Automated Surface Observing System (ASOS) wind measurements from 2007 to 2014. Wind and gust observations reported in the standard hourly ASOS dataset are shown to contain substantial bias caused by sampling and reporting protocols that restrict the reporting of gusts to arbitrarily defined "gusty" periods occurring during small subsets of each hour. The hourly ASOS gust reports are found to be inadequate for describing the gust characteristics of the site and ill suited for the study of gust factors. A gust-factor climatology was established for Milwaukee using the higherresolution, 1-min version of the ASOS dataset. The mean gust factor is 1.74. Stratified climatologies demonstrate that Milwaukee gust factors vary substantially with meteorological factors, with wind speed and wind direction exerting the strongest controls. A variety of modified gust-factor models were evaluated in which the peak wind gust is estimated by multiplying a gust factor by the observed, rather than forecast, wind speed. Errors thus obtained are entirely attributable to utility of the gust factor in forecasting peak gusts, having eliminated any error associated with the wind speed forecast. Results show that gust-factor models demonstrate skill in estimating peak gusts and improve with the use of meteorologically stratified gust factors.
\end{abstract}

\section{Introduction}

Wind gusts, sudden brief increases in wind speed (Huschke 1959), are accompanied by pressure and force fluctuations that are relevant to a diverse range of applications including wind energy; aviation; timber damage assessment; design codes for buildings, bridges, and electrical transmission lines; and resuspension models for deposited radioactive particles (Manasseh and Middleton 1999; Jung et al. 2016; Paulsen and Schroeder 2005; Giess et al. 1997; Pryor et al. 2014; Greenway 1979; Suomi et al. 2013; Wong and Miller 2010). Meteorological factors such as stability and turbulence exert strong influences, but gusts and wind speed are also controlled by nonatmospheric parameters that are termed metadata (Powell et al. 1996). Chief among these are averaging period and sampling duration, although instrument response characteristics, measurement height, and the height and spatial density of upwind terrain elements are also important (Jung and Schindler 2016; Mitsuta and Tsukamoto 1989; Carter 1974; Wichers Schreur and Geertsema 2008; Greenway

Corresponding author: Jonathan Kahl, kahl@uwm.edu
1979; Harper et al. 2010; Masters et al. 2010; Pryor et al. 2014; Suomi et al. 2016; Van der Hoven 1957).

The World Meteorological Organization (WMO) has provided recommendations for the precise definitions and measurement protocols of both wind gust and wind speed (Beljaars 1987). Worldwide consensus is lacking, however, and not all governmental agencies have adopted these guidelines. The WMO standard for estimating the wind speed is the 10-min average (Harper et al. 2010), yet the wind speed reported by the U.S. Automated Surface Observing System (ASOS) is a 2-min average (NOAA 1998). Wind gusts have been formally defined as "the wind speed deviation from the mean which, on average, is exceeded once during the reference period" (Kristensen et al. 1991). The WMO recommends defining a gust as the maximum 3 -s average during a 10-min sampling period (Suomi et al. 2015), but reporting requirements exist in some regions but not in others (Harper et al. 2010). The ASOS gust-reporting criteria, for example, include the requirement that gusts must exceed lulls by at least $10 \mathrm{kt}\left(1 \mathrm{kt}=0.51 \mathrm{~m} \mathrm{~s}^{-1}\right)$ (http:// w1.weather.gov/glossary/index.php?word=gust; http://w1. weather.gov/glossary/index.php?word=wind + gust).

The gust factor (GF) is the ratio of the wind gust to the wind speed. When averaged over extended time periods, 
it becomes a climatological measure of gustiness (Sherlock 1952). Gust factors figure prominently in wind conversion, the practice of converting between wind speeds averaged over different time periods (Harper et al. 2010), often for the purpose of standardizing or comparing wind impacts. Wind conversion is commonly used in applications relating to the intensity of tropical cyclones, structural engineering, and wind energy (Masters et al. 2010; Greenway 1979; Suomi et al. 2013). In the literature on tropical cyclones, the GF is defined as a theoretical conversion between a wind speed estimate and the expected highest gust of a given duration within a stated observation period (Harper et al. 2010). Other applications of wind conversion include exposure, a practice in which measured surface winds are corrected for the influence of flow obstacles (Powell et al. 2004). The determination of extreme winds, often defined as the maximum 10-min-averaged wind with a return period of $50 \mathrm{yr}$ (Lombardo et al. 2009; Larsén et al. 2012), is another application of wind conversion that is used by structural designers to estimate maximum wind loads.

Wind gust and wind speed metadata are clearly specified for certain wind-conversion applications. Conversion factors (i.e., gust factors) recommended for tropical-cyclone conditions assume gust durations of 3 , $60,120,180$, or $600 \mathrm{~s}$ and (mean) wind averaging periods of $60,120,180,600$, or $3600 \mathrm{~s}$ (Harper et al. 2010). For exposure estimation, Masters et al. (2010) standardized gust duration and wind averaging period at $3 \mathrm{~s}$ and $1 \mathrm{~h}$, respectively. Corresponding values of $10 \mathrm{~min}$ and $1 \mathrm{~h}$ are used for structural-design considerations (Soe and Thant 2014), whereas ASOS gusts are the highest 3-s wind measured during a 10 -min period.

Issues around sampling duration and averaging period notwithstanding, forecasting peak gusts remains difficult given the small spatial and temporal scales at which they occur (Mitsuta and Tsukamoto 1989). Although a variety of approaches have been developed, often on the basis of postprocessing of numerical weather prediction (NWP) model products (Suomi et al. 2013), no common parameterization exists to simulate wind gusts in NWP or regional climate models (Goyette et al. 2003). Parameterization methods include physical models that are based on downmixing of winds using model-generated soundings (Hart and Forbes 1999; Green and Poremba 2012), turbulence parameterizations (Panofsky and Dutton 1984; Schulz 2008; Wichers Schreur and Geertsema 2008; Brasseur 2001), and statistical methods involving model output statistics (Rudack 2006) and probability density functions (Friederichs et al. 2009).

The GF model is a simple statistical technique in which a known gust factor is multiplied by a forecast wind speed to yield a predicted peak wind gust. Comparisons among gust-modeling techniques, while scarce, suggest that the performance of GF models is comparable to that of other modeling approaches (Brasseur 2001; Goyette et al. 2003).

In addition to sampling and averaging protocols, gust factors are sensitive to meteorological and environmental factors including wind speed, stability, and upstream surface roughness (Davis and Newstein 1968; Carter 1974; Ágústsson and Ólafsson 2004; Shellard 1965; Harper et al. 2010; Masters et al. 2010; Suomi et al. 2013; Paulsen and Schroeder 2005). Typical values range from 1.3 over open water to 2.3 in the middle of large cities (Wieringa 1973; Harper et al. 2010; Pryor et al. 2014). Some studies have found tropical values to be generally larger than extratropical values (Choi and Hidayat 2002; Orwig and Schroeder 2007; Paulsen and Schroeder 2005). The GF models are in operational use by federal weather forecasting agencies in several countries, including the United States, United Kingdom, and Denmark (Cook et al. 2008; Kramer and Alsheimer 2013; Blaes et al. 2014; Woetmann Nielsen and Petersen 2001). It does not appear, however, that these operational GF models exploit the known sensitivities of the GF to changing meteorological conditions.

The two principal research questions addressed in this paper are whether the ASOS dataset is suitable for determining GF climatologies and whether the known sensitivities of the GF to meteorological variables can be exploited to improve GF model performance. To address these questions, we establish a GF climatology for Milwaukee, Wisconsin, and assess the GF-model performance at that location. We examine the sensitivity of GFs to several meteorological variables, and, unlike in previous studies, we harness these sensitivities by developing and evaluating meteorologically stratified GF models. Another novelty of this work is the adoption of a GF-model evaluation procedure in which gust forecasts are evaluated independent of the errors associated with wind speed forecasts. In addition, by including a limited analysis of GFs at another midlatitude site, we offer evidence that our method for developing and evaluating GF models can be used generally.

\section{Data and methods}

\section{a. Meteorological data}

ASOS wind speed, wind gust, wind direction, and cloud observations were obtained from the U.S. National Centers for Environmental Information (formerly National Climatic Data Center) for the period from January 2000 to December 2014 at Milwaukee (station 


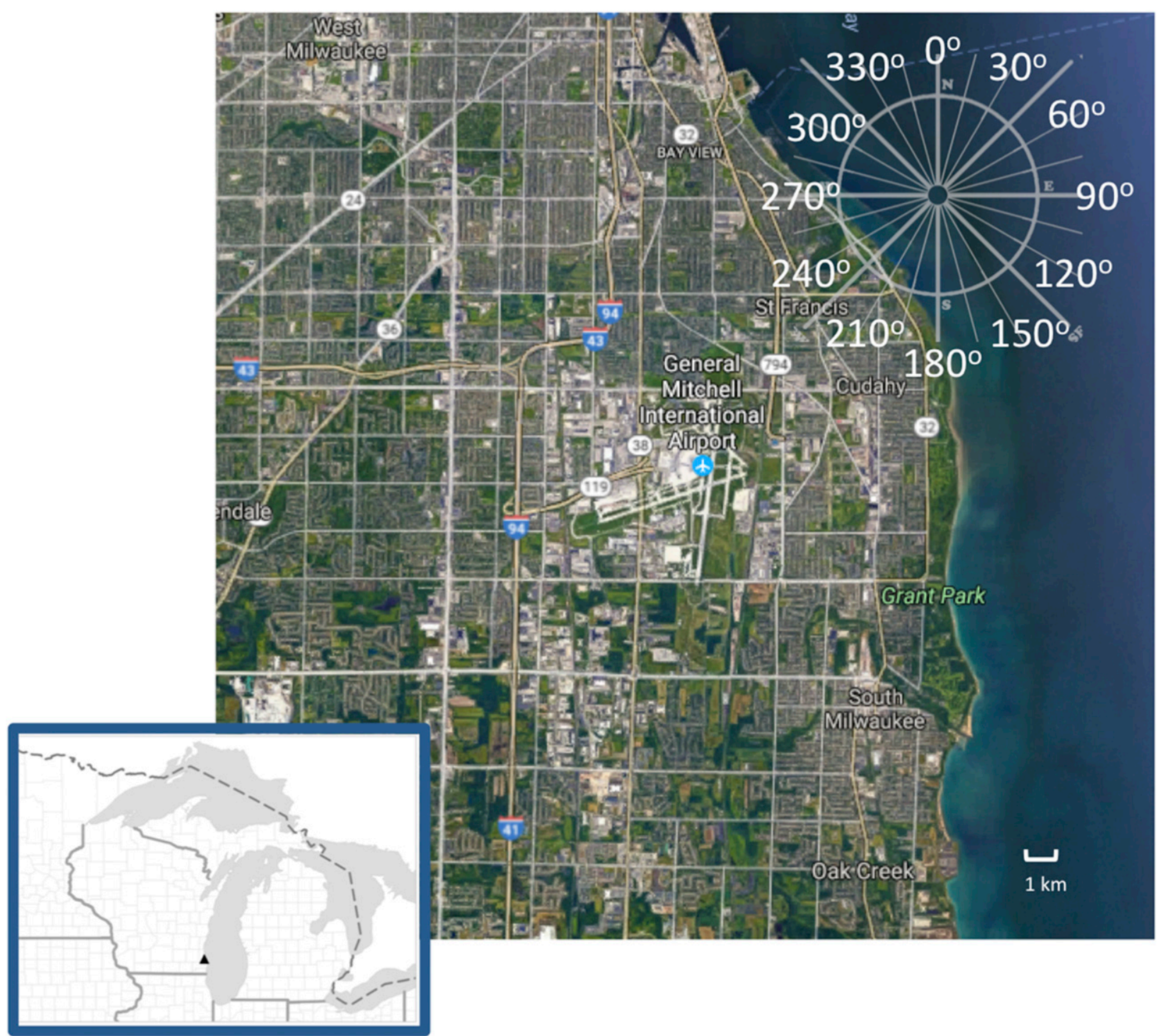

FIG. 1. Aerial view of the KMKE ASOS station, located at General Mitchell International Airport in Milwaukee.

identifier KMKE). Located at General Mitchell International Airport $\left(42.955^{\circ} \mathrm{N}, 87.904^{\circ} \mathrm{W} ; 204 \mathrm{~m} \mathrm{MSL}\right)$, KMKE is situated approximately $10 \mathrm{~km}$ south of downtown Milwaukee and $5 \mathrm{~km}$ west of Lake Michigan (Fig. 1). The ASOS observations are available hourly (NOAA 1998) and also with 1-min resolution at selected sites, including KMKE. In this study, both the standard hourly (denoted $\mathrm{ASOS}_{h}$ ) observations and the higherresolution, 1-min observations (denoted $\mathrm{ASOS}_{m}$ ) were used so as to examine the sensitivity of the GF to the observations from which they are derived.

Wind measurements at KMKE were made atop a 10-m tower. On 14 September 2006, the previously used Belfort Instrument Co. model 2000 cup and vane anemometer that reported 5-s-duration gusts was changed to a Vaisala, Inc., model 425 sonic anemometer reporting 3-s gusts (http://www.ncdc.noaa.gov/homr). Previous studies have shown that 3 -s gusts may be up to $5 \%$ larger than 5-s gusts (Lombardo et al. 2009; Suomi et al. 2015). This specific change in instrumentation has been shown to cause abrupt increases of $7 \%$ in gust factors (Masters et al. 2010). For these reasons, we restricted our analysis to the 2007-14 period.

In addition, $\mathrm{ASOS}_{m}$ and $\mathrm{ASOS}_{h}$ wind observations for 2014 were obtained for the ASOS station located in Atlanta, Georgia $\left(33.630^{\circ} \mathrm{N}, 84.442^{\circ} \mathrm{W} ; 308 \mathrm{~m} \mathrm{MSL}\right)$. Portions of the analyses conducted for Milwaukee were also performed for the shorter Atlanta dataset to demonstrate the applicability of the GF-model results to other locations. 
TABLE 1. Details of the ASOS wind and gust datasets.

\begin{tabular}{lcccc}
\hline \hline \multicolumn{1}{c}{ Dataset } & Abbreviation & Obs period & No. of hourly obs & No. of matching hourly obs $^{\mathrm{a}}$ \\
\hline ASOS hourly & ASOS $_{h}$ & Jan 2007-Dec 2014 & 9154 & 8495 \\
ASOS 1 min & ASOS $_{m}$ & Jan 2007-Dec 2014 & 61978 & 8495 \\
\hline
\end{tabular}

${ }^{\text {a }}$ Hours for which wind and gust observations were reported in both the $\mathrm{ASOS}_{h}$ and $\mathrm{ASOS}_{m}$ datasets.

\section{1) ONE-Minute $\left(\mathrm{ASOS}_{m}\right)$ OBSERVATIONS}

The $\mathrm{ASOS}_{m}$ wind and gust observations are the average and highest, respectively, 3-s wind observed each minute (NOAA 1998). After applying quality-control procedures to remove erroneous data, $61978 \mathrm{~h}$ of wind and gust observations were available for analysis, accounting for $88 \%$ of all hours during the 2007-14 study period (Table 1). [In the 2014 Atlanta $\mathrm{ASOS}_{m}$ dataset, $8142 \mathrm{~h}(92.9 \%)$ were available for analysis.]

\section{2) ONE-HOUR ( $\left.\operatorname{ASOS}_{h}\right)$ OBSERVATIONS}

The hourly wind speed reported in the $\mathrm{ASOS}_{h}$ dataset, hereinafter called the reported wind, is defined as the 2-min average of the 3-s wind measured during minutes 51 and 52 of the hour. The $\operatorname{ASOS}_{h}$ reported wind thus excludes observations made during $58 \mathrm{~min}(97 \%)$ of each hour. The $\mathrm{ASOS}_{h}$ gust observation, hereinafter called the reported gust, is defined as the highest 3-s average wind recorded during the 10-min period from minutes 43 to 52 of each hour. The reported gust thus excludes observations made during $50 \mathrm{~min}(83 \%)$ of each hour. Reported gusts must satisfy three criteria corresponding to minutes 43-52 of the hour: 1) the reported wind must be greater than $2 \mathrm{kt}, 2)$ the difference between the reported gust and the reported wind must be $\geq 3 \mathrm{kt}$, and 3 ) the reported gust must exceed the minimum 3 -s wind speed by at least $10 \mathrm{kt}$ (NOAA 1998).

The $\mathrm{ASOS}_{h}$ sampling protocols and reporting criteria create three important differences between gusts reported in the $\mathrm{ASOS}_{m}$ and $\mathrm{ASOS}_{h}$ datasets:

1) Gusts less than $14 \mathrm{kt}\left(7.2 \mathrm{~m} \mathrm{~s}^{-1}\right)$ are never reported in the hourly $\mathrm{ASOS}_{h}$ dataset.

2) The availability of $\mathrm{ASOS}_{h}$ gusts is limited by the reporting criteria. During the 2007-14 study period in Milwaukee, gusts were reported during $9154 \mathrm{~h}$, only $13.1 \%$ of all possible hours (Table 1 ). (In the 2014 Atlanta $\mathrm{ASOS}_{h}$ dataset, the corresponding percentage was $12.6 \%$.)

3) For any given hour, $\mathrm{ASOS}_{h}$ reported gusts are never larger than $\mathrm{ASOS}_{m}$ gusts. In section 3a, we show that the $\mathrm{ASOS}_{h}$ reported gusts are usually less than $\mathrm{ASOS}_{m}$ gusts as a result of the differences in observation periods (10 and $60 \mathrm{~min}$ for $\mathrm{ASOS}_{h}$ and $\mathrm{ASOS}_{m}$ gusts, respectively).

\section{b. Methods}

\section{1) Determining GuSt FACTORS}

Gust factors were determined using both the $\mathrm{ASOS}_{m}$ and $\mathrm{ASOS}_{h}$ observations. For the $\mathrm{ASOS}_{h}$ observations, calculating the GF $\left(\mathrm{GF}_{h}\right)$ is straightforward, because each hourly report, if the reporting criteria are met, contains only one wind observation and one gust observation:

$$
\mathrm{GF}_{h}=\frac{\text { reported gust }}{\text { reported wind }}
$$

The $\operatorname{ASOS}_{m}$ observations offer the opportunity to create a GF for each minute, but we calculate one GF per hour to allow for a more direct comparison with the $\mathrm{GF}_{h}$. We define a mean wind as the average of all sixty 1-min wind speed observations in the hour, and we define a peak gust as the highest 1-min gust observation in the hour (i.e., the highest 3-s wind speed during the entire hour). The GF from $\operatorname{ASOS}_{m}\left(\mathrm{GF}_{m}\right)$ is thus

$$
\mathrm{GF}_{m}=\frac{\text { peak gust }}{\text { mean wind }}
$$

The necessity of using the higher-resolution $\operatorname{ASOS}_{m}$ dataset in GF research has been noted before (e.g., Powell et al. 2004; Masters et al. 2010). Nevertheless, the $\mathrm{ASOS}_{h}$ dataset or its equivalent has been used in many GF studies (e.g., Brasseur 2001; Friederichs et al. 2009; Ágústsson and Ólafsson 2004; Green and Poremba 2012; Rudack 2006).

The effects of the $\mathrm{ASOS}_{h}$ sampling protocol on the determination of GFs are illustrated in Fig. 2, which shows $\mathrm{ASOS}_{m}$ and $\mathrm{ASOS}_{h}$ wind and gust observations for one sample hour in Milwaukee. In this example, the ASOS $_{m}$ peak gust (the highest wind speed observed during the entire hour) is $53 \mathrm{kt}\left(27.3 \mathrm{~m} \mathrm{~s}^{-1}\right)$, whereas the $\mathrm{ASOS}_{h}$ reported gust (the highest wind speed measured during the $10-\mathrm{min}$ period between minutes 43 and 52) is $41 \mathrm{kt}\left(21.1 \mathrm{~m} \mathrm{~s}^{-1}\right)$. (Note that the knot is the native unit of the ASOS wind instrumentation.) In a similar way, the $\mathrm{ASOS}_{m}$ mean wind (the average of all wind speed observations during the hour) is $27 \mathrm{kt}\left(13.9 \mathrm{~m} \mathrm{~s}^{-1}\right)$, whereas the $\mathrm{ASOS}_{h}$ reported wind (the average wind speed during the 2-min period between minutes 51 and 52) 


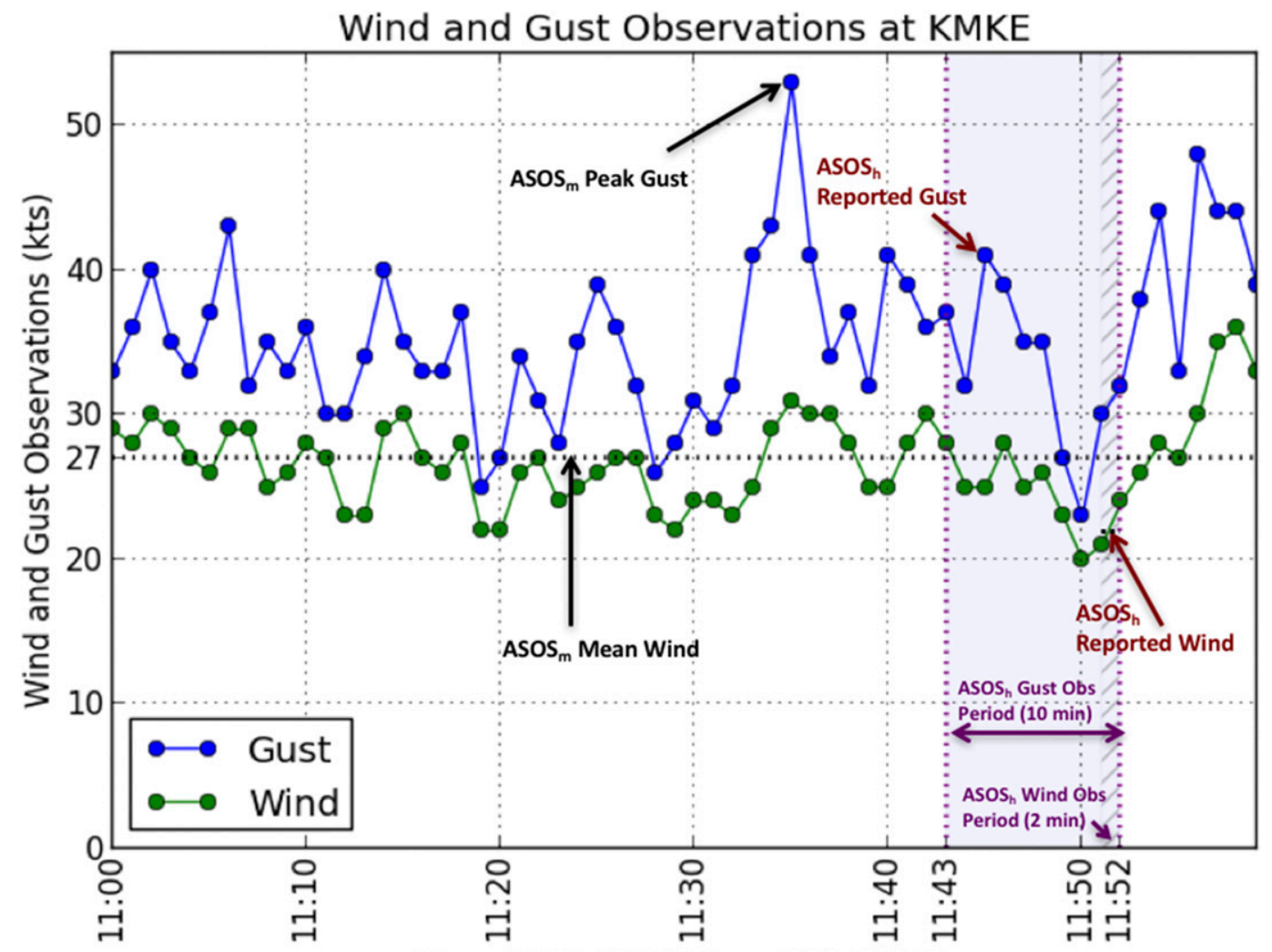

Time (CST; HH:MM) on 10/26/2010

FIG. 2. One-minute $\mathrm{ASOS}_{m}$ wind (green) and gust (blue) observations for one sample hour in Milwaukee, illustrating the effects of ASOS reporting practices on gusts and gust factors. The $\mathrm{ASOS}_{m} \mathrm{GF}$ [GF $\mathrm{GF}_{m}=$ (peak gust)/ $($ mean wind $)=1.96]$ incorporates observations during the entire hour, whereas the $\mathrm{ASOS}_{h} \mathrm{GF}\left[\mathrm{GF}_{h}=(\right.$ reported gust $) /($ reported wind $)=1.86$ ] is derived from observations during a 10-min period (purple shading), ignoring the majority of the hour's measurements.

is $22 \mathrm{kt}\left(11.3 \mathrm{~m} \mathrm{~s}^{-1}\right)$. The resulting GF using $\operatorname{ASOS}_{m}$ data is $\mathrm{GF}_{m}=1.96$, whereas that using $\mathrm{ASOS}_{h}$ data is $\mathrm{GF}_{h}=1.86$.

The differences in the periods for $\mathrm{ASOS}_{m}$ and $\mathrm{ASOS}_{h}$ wind and gust observations could thus result in sizeable differences between the $\mathrm{GF}_{m}$ and the $\mathrm{GF}_{h}$. Gust-factor differences of the magnitude shown in Fig. $2(0.10)$ are not negligible, given the range of typical values reported by Wieringa (1973). In section 3b, we present evidence that such differences are often exceeded in both the Milwaukee and Atlanta datasets.

As previously noted by Paulsen and Schroeder (2005) and Masters et al. (2010) in the context of tropicalcyclone winds, the use of $\mathrm{ASOS}_{h}$ data to determine GFs at a particular location may yield unrepresentative results, because this dataset reports only a small fraction of the wind observations made during each hour: $n=9154$ in the study presented here, as compared with $n=61978$ for the corresponding $\operatorname{ASOS}_{m}$ observations. In section $3 \mathrm{a}$, we show that the $\mathrm{ASOS}_{h}$ reporting criteria create an additional bias by including only stronger wind events. Because no standard protocol exists for the wind and gust averaging times used in the computation of gust factors (Davis and Newstein 1968; Harper et al. 2010), a key aspect of this work is to elucidate the influence of the $\mathrm{ASOS}_{h}$ reporting criteria and sampling periods on GFs and their subsequent climatologies.

\section{2) Meteorologically STRATIFIED GUST FACTORS}

In addition to elucidating the differences between the $\mathrm{GF}_{m}$ and the $\mathrm{GF}_{h}$, we create a GF climatology by stratifying the GFs according to wind speed, wind direction, season, month, time of day, and atmospheric stability. This stratification is motivated by a desire to better understand the meteorological controls on GFs, which in turn could prove useful in forecasting wind gusts. The stratification is applied only to $\mathrm{GF}_{m}$, because the $\mathrm{ASOS}_{m}$ dataset has the capability to better capture the character of wind gusts, as compared with the $\mathrm{ASOS}_{h}$ dataset, for 
which sampling protocols and reporting criteria create evident biases.

The $\mathrm{GF}_{m}$ GFs were stratified into 5-kt $\left(2.6 \mathrm{~m} \mathrm{~s}^{-1}\right)$ bins for wind speed and $30^{\circ}$ bins for wind direction. Gust-factor stratification by atmospheric stability was accomplished using the Pasquill (1961) stability classification scheme, which considers surface-based observations of cloud cover, insolation (incoming solar radiation), and wind speed. Following Luna and Church (1972), insolation was determined via a combination of cloud cover and solar angle. Sounding data were not used because of the absence of a collocated radiosonde station in Milwaukee, as well as the insufficiency of twice-daily radiosonde measurements in adequately describing diurnal cycles of stability.

\section{3) GuST-FACTOR MODELS}

The GF model

$$
\text { gust }_{\text {fcst }}=\mathrm{GF} \times \mathrm{wspd}_{\mathrm{fcst}}
$$

is a simple means of forecasting the peak gust (gust fcst $_{\text {) }}$ ) given a known GF and a forecast wind speed wspd $_{\text {fcst }}$, the latter often obtained from NWP models. Any error in the gust forecast thus includes a contribution from the error associated with the wind speed forecast. In this study, we replace wspd $_{\text {fcst }}$ with a wind speed observation corresponding to the forecast hour. This equates to making a perfect wind speed "forecast," eliminating any errors associated with $\operatorname{wspd}_{\text {fcst }}$. With this strategy, the skill of the GF model in Eq. (3) is solely due to the representativeness of the GF that is used.

Wind gust forecasts were evaluated for all hours in the 2007-14 period for which wind and gust observations were available. Forecast performance was also evaluated for "gusty" subsets of the overall period, during which observed gusts exceeded $25 \mathrm{kt}\left(12.9 \mathrm{~m} \mathrm{~s}^{-1}\right)$.

Several variants of the GF in Eq. (3) were used. MeanGF models used the average $\mathrm{GF}_{m}$ and $\mathrm{GF}_{h}$ corresponding to the entire study period. These values are reported in section $3 \mathrm{~b}$. Stratified models incorporated $\mathrm{GF}_{m}$ stratified by wind speed [5-kt $\left(2.6 \mathrm{~m} \mathrm{~s}^{-1}\right)$ bins], wind direction $\left(30^{\circ}\right.$ bins), stability (Pasquill category), time of day, and season. A doubly stratified GF model was also tested, using $\mathrm{GF}_{m}$ stratified by a combination of wind speed and wind direction. All GF models were compared with the no-skill models of "persistence" (gust $_{\mathrm{fcst}}=$ gust observation from previous hour) and

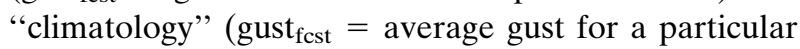
season and time of day).

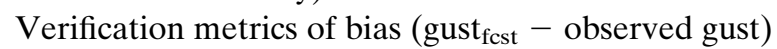
and absolute error (|bias $\mid)$ were used for all models. Mean- and stratified- $\mathrm{GF}_{m}$ models were verified against the $\mathrm{ASOS}_{m}$ peak-gust observations, and the mean- $\mathrm{GF}_{h}$ model was verified against the $\mathrm{ASOS}_{h}$ reported gust observations. The $\mathrm{GF}_{h}$ model was also verified against the $\operatorname{ASOS}_{m}$ peak-gust observations to illustrate the consequences of both formulating and evaluating GF models using observations corresponding to only small subsets of each hour. Absolute-error distributions for selected pairs of models were compared via the sign test (Mendenhall et al. 1990) and were considered to be statistically significant when the null hypothesis (of no difference) was rejected at the $99 \%$ confidence level.

\section{Results and discussion}

\section{a. Wind and gust climatology}

The gust factor as defined in Eqs. (1) and (2) is the ratio of an observed wind gust to an observed wind speed. Both numerator and denominator are sensitive to the sampling periods and reporting criteria that are associated with the datasets from which they are derived. Therefore, before presenting the GF climatology, we first examine these sensitivities.

The sensitivity of wind speed observations to the gustreporting criteria is shown in Fig. 3a. The gust-reporting criteria ( $\mathrm{ASOS}_{h}$ dataset only) severely reduce the number of observations and strongly bias the distribution toward higher wind speeds. Climatological GFs determined using the $\mathrm{ASOS}_{h}$ dataset would therefore be expected to be smaller than those determined with $\operatorname{ASOS}_{m}$ data, because the wind speeds (the GF denominator) are larger.

Distributions of $\mathrm{ASOS}_{m}$ mean wind (60-min average) and $\mathrm{ASOS}_{h}$ reported wind (2-min average) during hours for which a gust is reported in both datasets-thereby removing the bias due to gust-reporting criteria-are remarkably similar (Fig. 3b). This is not surprising, given the known gap in the wind speed energy spectrum occurring between periods of a few minutes and a few hours (Van der Hoven 1957), although subsequent analyses suggest that the spectral gap may be only a factor of 2 lower than the higher-frequency peak (Jensen 1999).

As expected, $\mathrm{ASOS}_{h}$ gust observations are also highly sensitive to the reporting criteria, as shown in Fig. 3c. Recall from section 2a(2) that $\mathrm{ASOS}_{h}$ gusts are reported only when they exceed the $\mathrm{ASOS}_{h}$ reported wind, as well as lulls, by specified amounts. Like the case for wind speed, the reporting criteria severely reduce the number of $\mathrm{ASOS}_{h}$ reported gusts and bias the observations toward higher values. The lack of such criteria for the $\mathrm{ASOS}_{m}$ peak gusts explains the occasional presence of very weak "gusts" [i.e., $\left.<5 \mathrm{kt}\left(2.6 \mathrm{~m} \mathrm{~s}^{-1}\right)\right]$. Unlike the wind speed observations, however, gust observations from the two datasets exhibit an important sensitivity to 

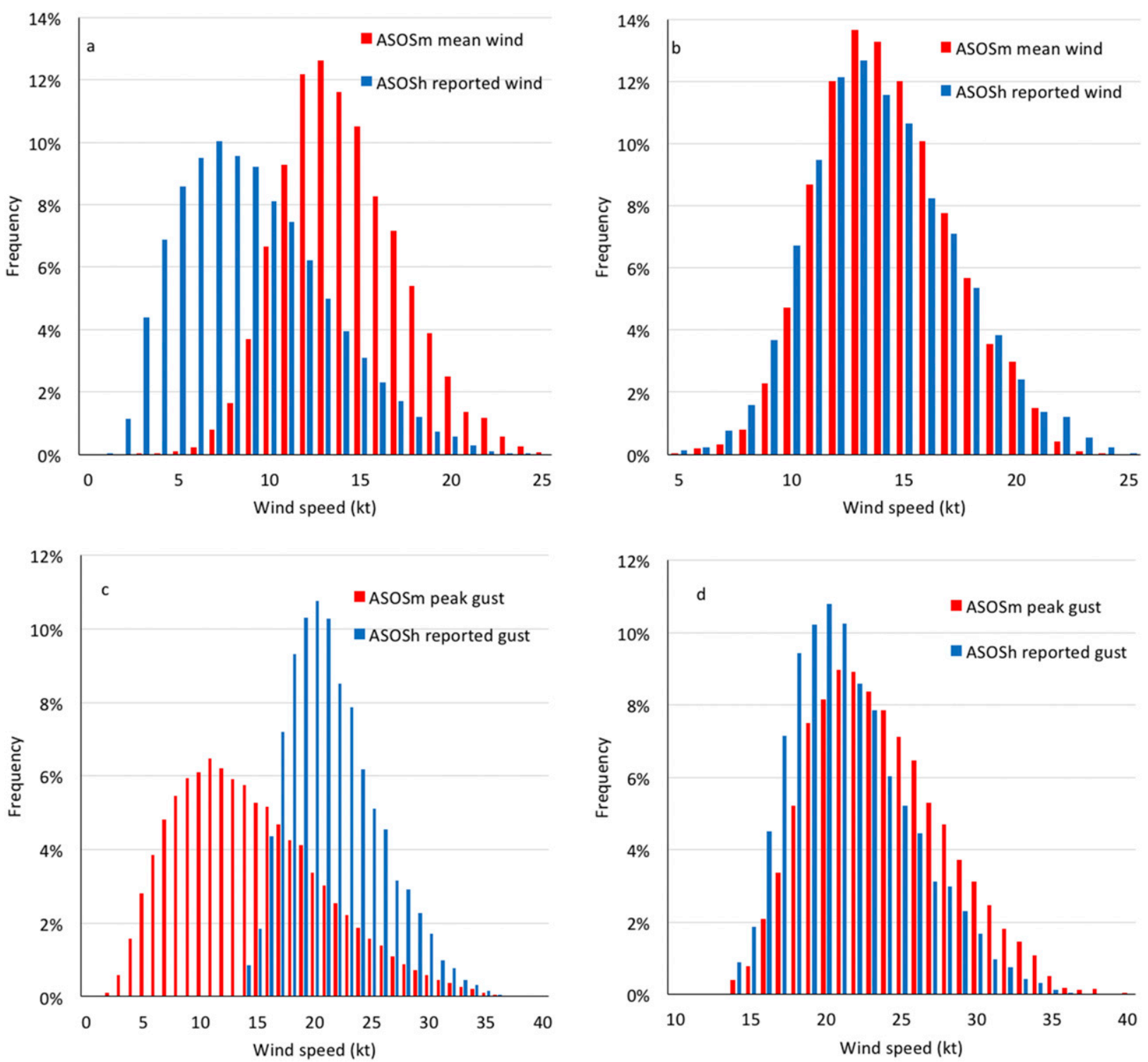

FIG. 3. Wind and gust observations during 2007-14 in Milwaukee, illustrating sensitivity to data artifacts. (a) Distributions of ASOS $m$ mean wind (red) and $\mathrm{ASOS}_{h}$ reported wind (blue), illustrating sensitivity to $\mathrm{ASOS}_{h}$ gust-reporting criteria. (b) Distributions of ASOS ${ }_{m}$ mean wind (red) and $\mathrm{ASOS}_{h}$ reported wind (blue), but with only hours for which gusts are reported in both datasets included. (c) Distributions of $\mathrm{ASOS}_{m}$ peak gusts (red) and $\mathrm{ASOS}_{h}$ reported gusts (blue), illustrating sensitivity to ASOS ${ }_{h}$ gust-reporting criteria. (d) Distributions of $\mathrm{ASOS}_{m}$ peak gust (red) and $\mathrm{ASOS}_{h}$ reported gust (blue), but with only hours for which gusts are reported in both datasets included, illustrating sensitivity to $\mathrm{ASOS}_{h}$ sampling period.

sampling periods (Fig. 3d). During hours for which both are reported, the $\mathrm{ASOS}_{m}$ peak gust is always greater than or equal to the $\mathrm{ASOS}_{h}$ reported gust. Although more than half of the differences ASOS $_{m}$ peak gust minus $\mathrm{ASOS}_{h}$ reported gust) are under $5 \mathrm{kt}\left(2.6 \mathrm{~m} \mathrm{~s}^{-1}\right)$ when $\mathrm{ASOS}_{h}$ gusts are reported, the differences become larger with increasing gustiness (Fig. 4), because the hourly $\mathrm{ASOS}_{h}$ gusts sample only $10 \mathrm{~min}$ of each hour Differences of 5 and $10 \mathrm{kt}\left(2.6\right.$ and $\left.5.1 \mathrm{~m} \mathrm{~s}^{-1}\right)$ or larger were found in $11.8 \%$ and $0.8 \%$ of observations, respectively.

The data artifacts resulting from reporting criteria and sampling periods thus render the $\mathrm{ASOS}_{h}$ wind and gust data ill suited for the determination of GFs, which are intended to be representative measures of local climatological gustiness (Sherlock 1952). The $\mathrm{ASOS}_{m}$ observations, which better capture the character of wind gusts, are a much more appropriate means of determining GFs. 


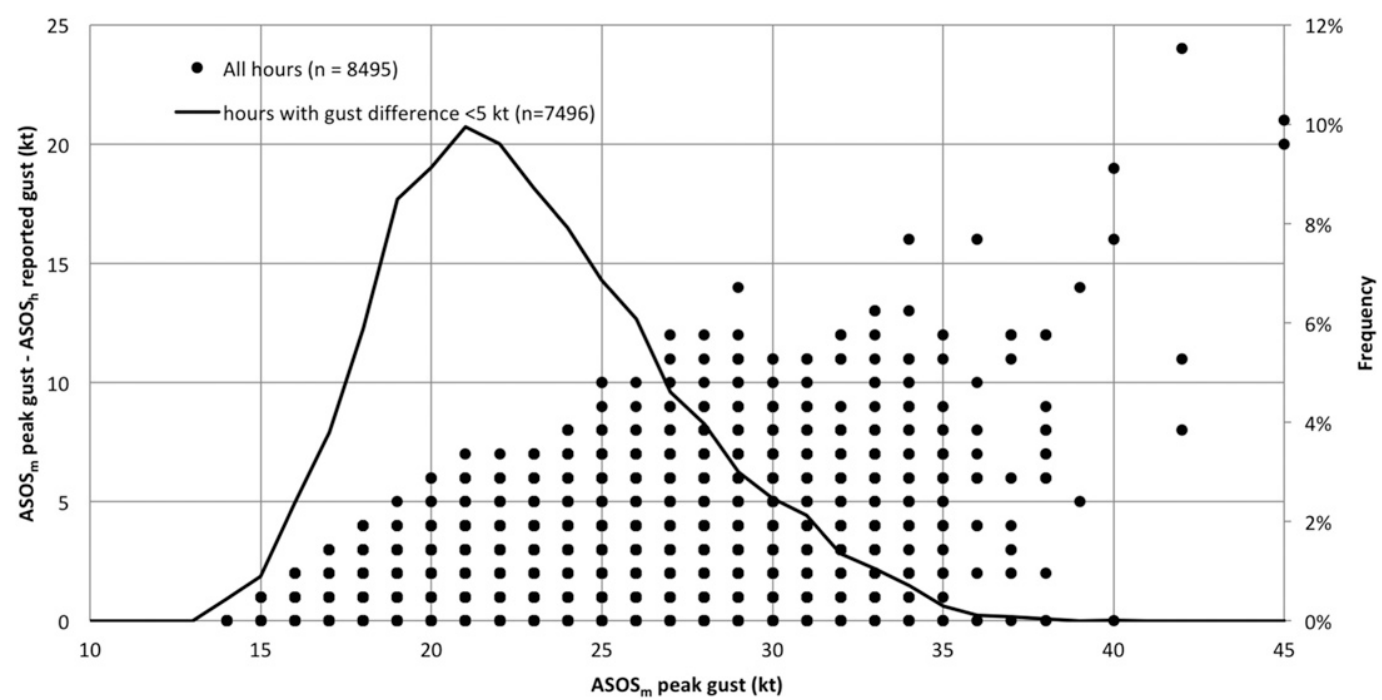

FIG. 4. Difference in gust observations $\left(\mathrm{ASOS}_{m}\right.$ peak gust minus $\mathrm{ASOS}_{h}$ reported gust) as a function of $\mathrm{ASOS}_{m}$ peak gust during 2007-14 in Milwaukee during hours for which a gust is reported in both datasets $(n=8495)$. Each circle represents multiple points, because gusts from both datasets are reported in integer units. The curve (right scale) shows the frequency distribution of the strength of $\operatorname{ASOS}_{m}$ peak gusts for hours in which gust differences are $<5 \mathrm{kt}\left(2.6 \mathrm{~m} \mathrm{~s}^{-1}\right)$.

(Refer to the appendix for additional evidence of the biases contained in the $\mathrm{ASOS}_{h}$ dataset.)

\section{b. Gust-factor climatology}

The mean $\mathrm{GF}_{m}$ for Milwaukee is 1.74, as based on the full set of 1-min $\operatorname{ASOS}_{m}$ data during 2007-14 ( $n=$ 61978). The corresponding mean $\mathrm{GF}_{h}$, as based on the full set of hourly $\mathrm{ASOS}_{h}$ data during the same period $(n=9154)$, is 1.58 . Similar values for extratropical locations were reported by Paulsen and Schroeder (2005) and Cook (1986).

The mean $\mathrm{GF}_{h}$ is $9.2 \%$ smaller than the mean $\mathrm{GF}_{m}$, confirming the expected low bias associated with the $\mathrm{ASOS}_{h}$ gust-reporting criteria and sampling protocols. [In Atlanta, the mean $\mathrm{GF}_{h}(1.65 ; n=1106)$ is $13.6 \%$ smaller than the mean $\mathrm{GF}_{m}(1.91 ; n=8142)$.] When we eliminate the $\mathrm{ASOS}_{h}$ reporting bias by considering only hours for which gusts are reported in both datasets $(n=8495)$, the mean difference $\mathrm{GF}_{m}-$ $\mathrm{GF}_{h}$ is 0.15 (Fig. 5). This difference again emphasizes the pervasive impact of the sampling period on the determination of GFs: even without the low bias due to the $\mathrm{ASOS}_{h}$ gust-reporting criteria, sampling gusts during only $10 \mathrm{~min}$ of each hour causes a low bias of $9 \%$ in GFs calculated using this dataset. Having demonstrated that GFs that are based on $\mathrm{ASOS}_{h}$ data suffer from artifacts due to sampling and reporting protocols, the remaining climatology of gust factors stratified by wind speed and other variables will be restricted to the $\mathrm{GF}_{m}$ gust factors, which are based on $\operatorname{ASOS}_{m}$ data.

Gust factors are known to be sensitive to surface roughness (Powell et al. 2004; Pryor et al. 2014; Suomi et al. 2013), although Harper et al. (2010) point out that GFs determined for sites located on hills or slopes may not be representative of their true gust climates because of changing wind direction and/or the lack of an extended constant roughness fetch for many kilometers. Masters et al. (2010) provided a database of directionally stratified GFs and surface-roughness values for hurricane-prone regions in the eastern United States, but because their focus was on tropical-storm conditions their method excluded conditions with mean winds under $5 \mathrm{~m} \mathrm{~s}^{-1}(9.7 \mathrm{kt})$ and nonneutral stability-roughly one-third of their dataset.

In this study, wind direction was used as a proxy for surface roughness because the measurement site is surrounded by highly heterogeneous terrain (Fig. 1). Wind directions from south-southeast to north and from north-northeast to southeast have large fetches over land and water, respectively. Wind direction had a sizeable impact on the $\mathrm{GF}_{m}$ (Fig. 6). When one considers all wind speeds together, mean $\mathrm{GF}_{m}$ values of 1.6-1.7 occurred with winds with a long fetch over Lake Michigan $\left(60^{\circ}-150^{\circ}\right)$ whereas larger values up to 1.8 were associated with inland winds $\left(240^{\circ}-30^{\circ}\right)$. Other studies have similarly reported higher GFs in association with larger surface roughness (Wieringa 1973; 


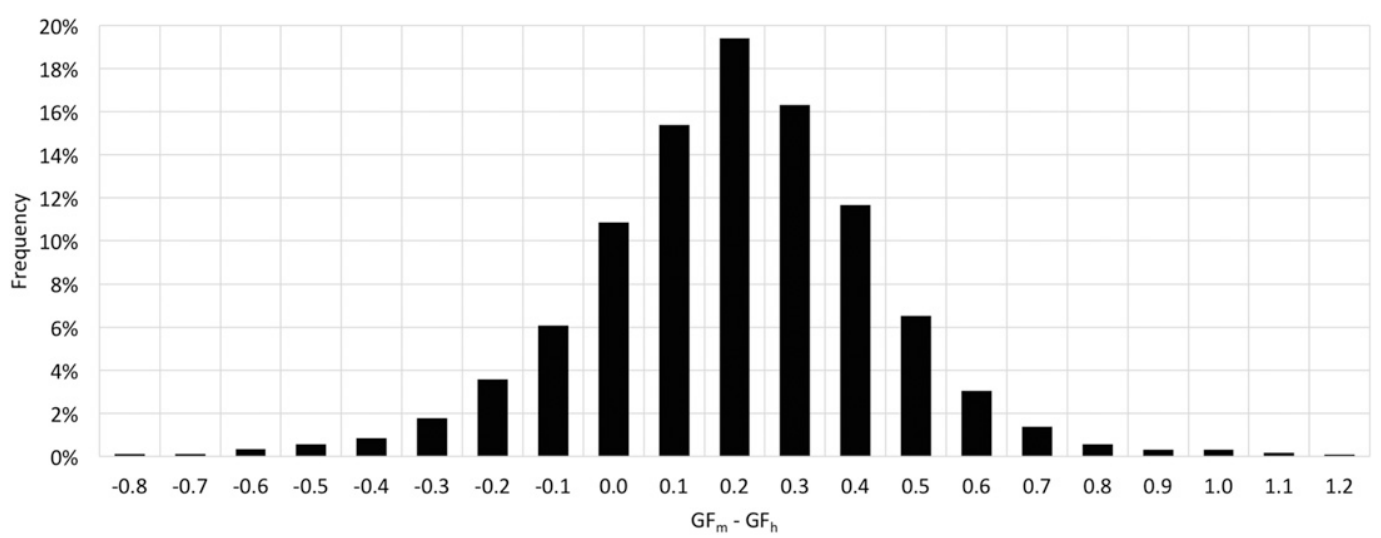

FIG. 5. Distribution of differences (kt) between $\mathrm{ASOS}_{m}$ and $\mathrm{ASOS}_{h}$ gust factors $\left(\mathrm{GF}_{m}-\mathrm{GF}_{h}\right)$ during 2007-14 in Milwaukee, illustrating sensitivity to sampling period. Only hours for which gusts are reported in both datasets are included.

Harper et al. 2010; Masters et al. 2010; Paulsen and Schroeder 2005; Powell et al. 2004).

Wind speed also exerts an important influence on GFs. Milwaukee GFs decrease with increasing wind speed (Fig. 6) - a finding that is consistent with previous studies (Davis and Newstein 1968; Carter 1974; Mitsuta and Tsukamoto 1989; Ágústsson and Ólafsson 2004; Paulsen and Schroeder 2005; Pryor et al. 2014). When one considers all wind directions together, the mean $\mathrm{GF}_{m}$ decreases from 1.91 to 1.64 when the wind speeds increase from the $0-5-\mathrm{kt}\left(0-2.6 \mathrm{~m} \mathrm{~s}^{-1}\right)$ range to $\geq 15 \mathrm{kt}\left(7.7 \mathrm{~m} \mathrm{~s}^{-1}\right)$. This GF sensitivity of 0.27 to differing wind speeds is meaningful, because it represents $27 \%$ of the range of typical values reported by Wieringa (1973).
The variability in the $\mathrm{GF}_{m}$ is largest when wind speeds are under $5 \mathrm{kt}\left(2.6 \mathrm{~m} \mathrm{~s}^{-1}\right)$, with standard deviations of $\sim 0.4$ regardless of wind direction (Fig. 6). This variability decreases as wind speeds increase, with standard deviations dropping to $\sim 0.15$ for winds in the $10-15-\mathrm{kt}$ $\left(5.1-7.7 \mathrm{~m} \mathrm{~s}^{-1}\right)$ range. In Milwaukee, $\mathrm{GF}_{m}$ variability is more sensitive to wind speed than to wind direction, though this result may depend somewhat on the definition of the wind speed ranges and the wind direction sectors utilized.

Previous studies have reported a relationship between GF and stability in which the GF decreases as the atmosphere becomes more stable (Carter 1974; Kramer and Alsheimer 2013; Davis and Newstein 1968; Ágústsson and Ólafsson 2004). In Milwaukee, we find

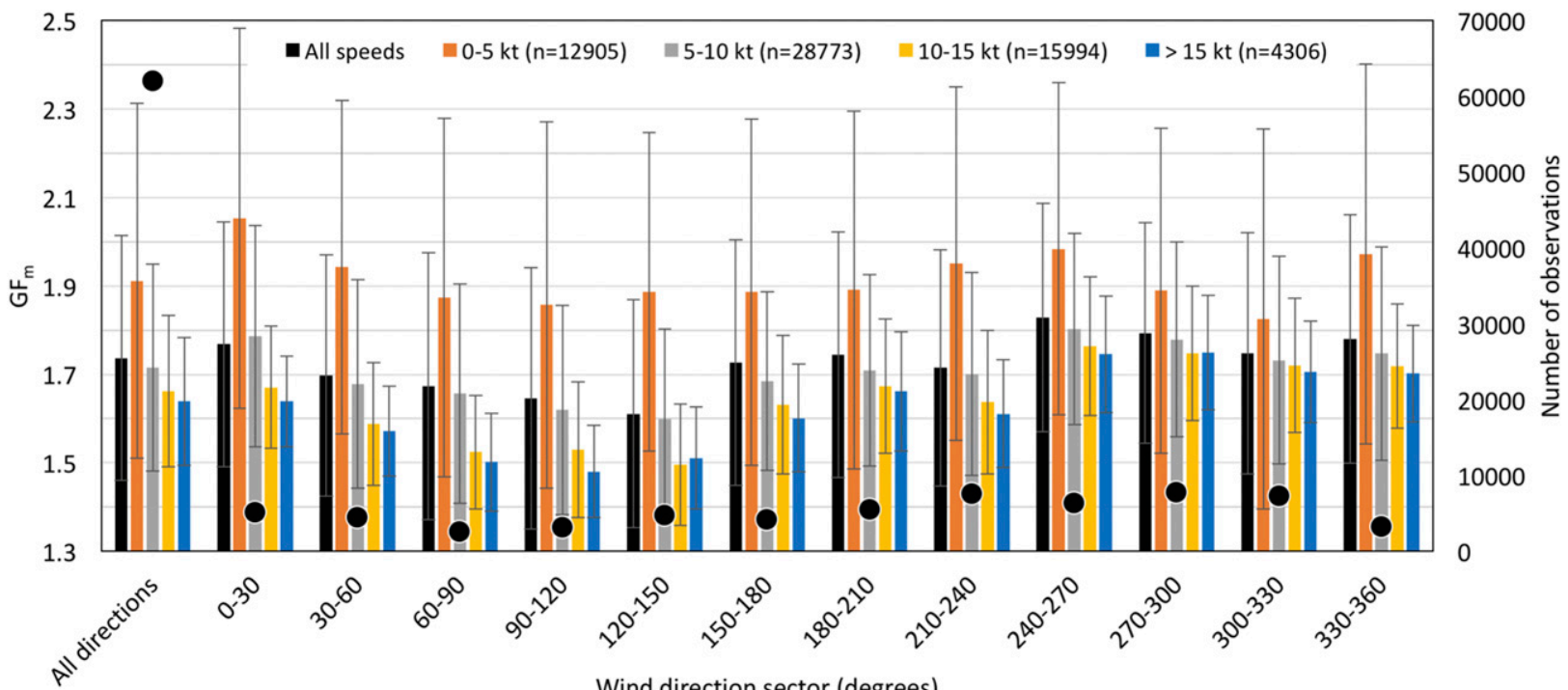

FIG. 6. $\operatorname{ASOS}_{m} \mathrm{GF}_{m}$ (bars; left scale) stratified by wind speed and direction over 2007-14. Error bars indicate \pm 1 standard deviation. The number of observations (circles; right scale) is shown for each wind direction sector. 


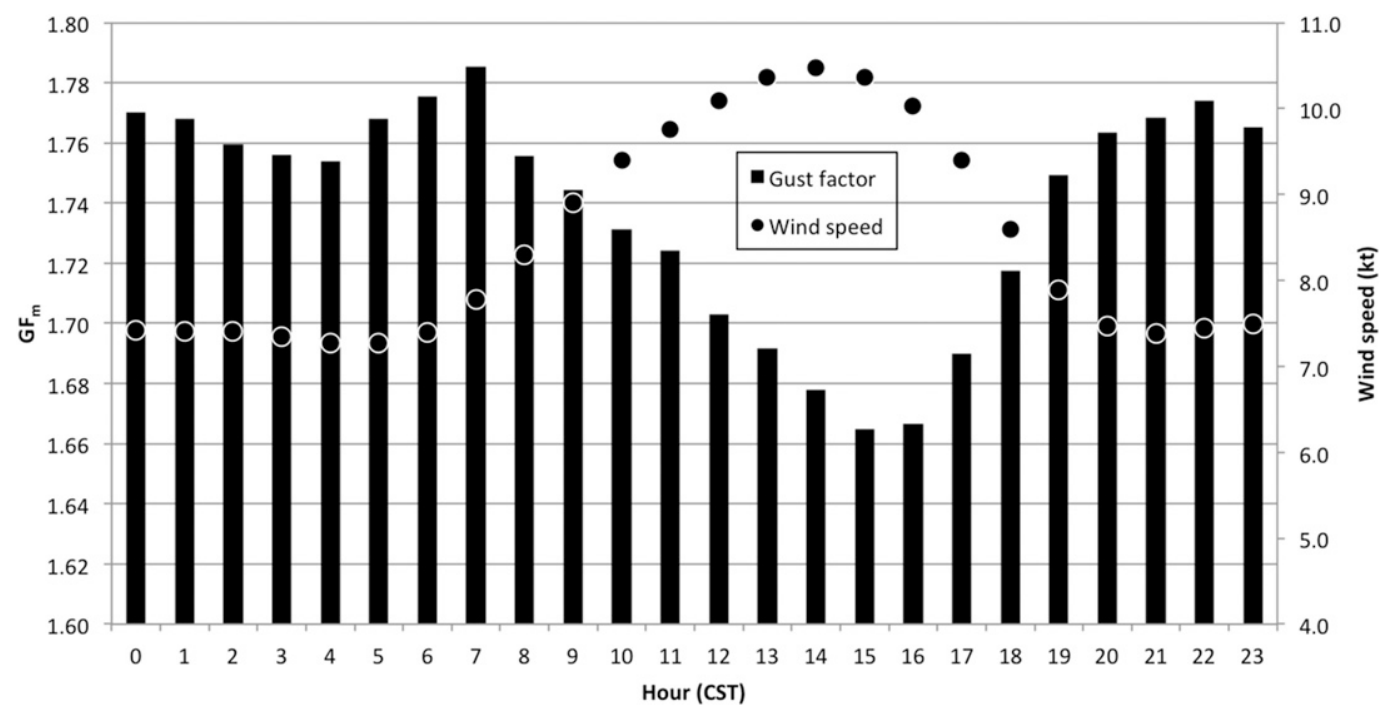

FIG. 7. The $\mathrm{GF}_{m} \mathrm{~s}$ (bars) and wind speed (circles) stratified by hour on the basis of Milwaukee $\mathrm{ASOS}_{m}$ data for 2007-14.

that the $\mathrm{GF}_{m}$ indeed decreases from 2.0 for "extremely unstable" conditions (Pasquill category A) to 1.68 for "neutral" conditions (category D), in agreement with the aforementioned previous studies. As stability increases further from the neutral (D) to the "stable" (F) categories, the $\mathrm{GF}_{m}$ does not continue to decrease but rather increases to 1.84 .

The stability-stratified GF results may be understood by considering the role of wind speed in determining stability categories (Pasquill 1961). During the daytime, an increase in categorical stability from unstable (A) to neutral (D) is associated with an increase in wind speed. At night, the opposite is true: a stability increase from neutral (D) to stable (F) is accompanied by a decrease in wind speed. The strong sensitivity of GF to wind speed that was noted earlier (Fig. 6) thus appears to partially mask the sensitivity of GF to stability.

A site-specific wind direction effect may also contribute to the lack of a monotonic dependence of GF on stability because in Milwaukee advection from the direction of Lake Michigan typically brings unstable air in the winter and stable air in the summer. In addition, the Pasquill classification scheme may not provide an adequate representation of atmospheric stability over the neutral-to-stable range (Luna and Church 1972).

In addition to being influenced by wind speed, wind direction, and stability, the $\mathrm{GF}_{m}$ exhibited moderate variation when stratified by hour of day (Fig. 7). The $\mathrm{GF}_{m}$ was lowest during the afternoon and highest around sunrise. This is consistent with the inverse relationship between $\mathrm{GF}_{m}$ and wind speed (Fig. 6), because wind speed (also shown in Fig. 7) peaks during the afternoon and reaches a minimum just before sunrise.

\section{c. Performance of the gust-factor model}

In this study, we replace $\operatorname{wspd}_{\text {fcst }}$ in the GF model of Eq. (3) with the observed wind speed during the forecast hour. Any error in gust $_{\mathrm{fcst}}$ is therefore attributable to the representativeness of the GF in forecasting peak gusts (i.e., in the GF model itself). Our model performance results thus represent a "best case scenario," because operational GF models will suffer from imperfect wind speed forecasts.

Results of GF-model performance for the full 2007-14 period are shown in Table 2. Verification metrics were determined for all models: mean (nonstratified) and stratified $\mathrm{GF}_{m}$ and $\mathrm{GF}_{h}$, persistence, and climatology. The type of observation used for verification is also listed in Table 2 ASOS $_{m}$ peak gust or $\mathrm{ASOS}_{h}$ reported gust). The mean absolute error (MAE) ranges from 1.20 to $4.82 \mathrm{kt}\left(0.62-2.48 \mathrm{~m} \mathrm{~s}^{-1}\right)$ over all models. The mean bias is small, between -0.12 and $0.62 \mathrm{kt}$ (between -0.06 and $0.32 \mathrm{~m} \mathrm{~s}^{-1}$ ), for all models except the $\mathrm{GF}_{h}$ model verified against the $\mathrm{ASOS}_{m}$ peak gust. The mean bias for this model is $-1.67 \mathrm{kt}\left(-0.86 \mathrm{~m} \mathrm{~s}^{-1}\right)$, reflecting the fact that the $\mathrm{ASOS}_{h}$ reported gusts can never be larger than the $\mathrm{ASOS}_{m}$ peak gusts.

The GF-model results are meaningful in a variety of contexts. Most important, as noted above, is that they represent an upper bound on the performance expected of an operational GF model. A sense of the additional error arising from imperfect wind speed forecasts may be obtained by sampling wind speed forecast errors from distributions reported in recent studies (Traiteur et al. 2012; Zamo et al. 2016; Cheng et al. 2017). Accordingly, wind speed forecast errors sampled from a distribution 
with MAE, mean bias, and standard deviation of $2.92 \mathrm{kt}$ $\left(1.5 \mathrm{~m} \mathrm{~s}^{-1}\right), 0.0 \mathrm{kt}\left(0.0 \mathrm{~m} \mathrm{~s}^{-1}\right)$, and $2.33 \mathrm{kt}\left(1.2 \mathrm{~m} \mathrm{~s}^{-1}\right)$, respectively, when added to wspd $_{\text {fcst }}$ in Eq. (3) would increase the MAE of a GF model by $2.16 \mathrm{kt}\left(1.11 \mathrm{~m} \mathrm{~s}^{-1}\right)$. This mean wind forecast error component is larger than the MAEs of most GF models shown in Table 2.

Although the MAE is fairly small-less than $3 \mathrm{kt}$ $\left(1.54 \mathrm{~m} \mathrm{~s}^{-1}\right)$ for nearly all GF models, there are many cases for which errors are much larger. The MAE for the mean $\mathrm{GF}_{m}$ model is $1.40 \mathrm{kt}\left(0.72 \mathrm{~m} \mathrm{~s}^{-1}\right)$, for example, but there are $1112 \mathrm{~h}$ during $2007-14$ (1.9\% of all hours analyzed) for which the absolute error exceeds $5 \mathrm{kt}$ $\left(2.6 \mathrm{~m} \mathrm{~s}^{-1}\right)$. These are typically hours for which particularly small or large GFs are observed (i.e., GF $<1.5$ or GF > 2.0). Moreover, large differences in model performance are evident at times. For example, there are $202 \mathrm{~h}$ (3.7\% of matched cases) when the difference in the absolute errors between the mean $\mathrm{GF}_{m}$ and mean $\mathrm{GF}_{h}$ models exceeds $5 \mathrm{kt}\left(2.6 \mathrm{~m} \mathrm{~s}^{-1}\right)$ and $25 \mathrm{~h}(0.45 \%)$ when the difference exceeds $8 \mathrm{kt}\left(4.1 \mathrm{~m} \mathrm{~s}^{-1}\right)$. These hours are typically characterized by large differences between $\mathrm{ASOS}_{m}$ and $\mathrm{ASOS}_{h}$ winds or gusts.

Even small performance differences among GF models may have both physical relevance and operational significance, because errors in gust forecasts may also include errors in wind speed estimation. In windenergy applications, for example, an important factor in selecting a location for turbine placement is the windenergy density, which is a measure of the total amount of power in a moving air mass and is proportional to the third power of the wind speed (Johnson and Erhardt 2016). Another example is building construction, in which wind loads on crane operations and partially completed structures may cause damage at speeds well below the design wind speed for the completed structure (Houghton 1985).

A further comparison of the mean $\mathrm{GF}_{m}$ and mean $\mathrm{GF}_{h}$ models lends insight into the impact of the restricted $\mathrm{ASOS}_{h}$ observation periods on GF model performance. The mean $\mathrm{GF}_{m}$ model exhibits an MAE of $1.40 \mathrm{kt}\left(0.72 \mathrm{~m} \mathrm{~s}^{-1}\right)$ (Table 2). The mean $\mathrm{GF}_{h}$ model, which is based on GFs derived from the $\mathrm{ASOS}_{h}$ dataset with its $2 \mathrm{minh}^{-1}$ wind observing period and 10-min observing period for gusts, exhibits a larger MAE of $2.49 \mathrm{kt}\left(1.28 \mathrm{~m} \mathrm{~s}^{-1}\right)$ when verified against $\operatorname{ASOS}_{h}$ reported gusts. The difference in the error distributions of these two models is statistically significant, providing evidence that GF models derived from the $\operatorname{ASOS}_{m}$ dataset perform better than models that are based on $\mathrm{ASOS}_{h}$ data. [In Atlanta, the mean $\mathrm{GF}_{m}$ model similarly outperformed the mean $\mathrm{GF}_{h}$ model, with MAEs of $1.97 \mathrm{kt}\left(1.01 \mathrm{~m} \mathrm{~s}^{-1}\right)$ and $3.27 \mathrm{kt}\left(1.68 \mathrm{~m} \mathrm{~s}^{-1}\right)$, respectively.] Moreover, the performance of the $\mathrm{GF}_{h}$ 


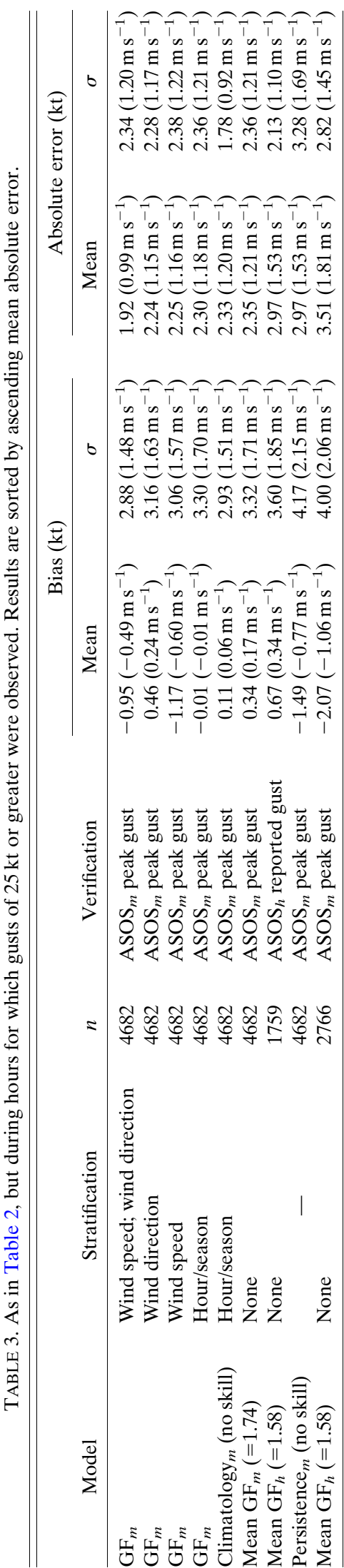

model is misleading. When verified against $\mathrm{ASOS}_{m}$ peak gusts, which describe the peak gust during the entire hour, the MAE increases by $22 \%$ to $3.05 \mathrm{kt}\left(1.57 \mathrm{~m} \mathrm{~s}^{-1}\right)$.

The mean $\mathrm{GF}_{h}$ model thus suffers from an ill-posed formulation in which 1) $\mathrm{GF}_{h}$ derived from wind and gust observations sampled during very small subsets of each hour is considered to be representative of the overall climatological gustiness, 2) these $\mathrm{GF}_{h} \mathrm{~s}$ are used in Eq. (3) to predict the maximum gust that will occur during the entire forecast hour, and 3) with the $\mathrm{ASOS}_{h}$ dataset, verification of gust $_{\text {fcst }}$ is only possible during the 10-min "reported gust" measurement period.

The $\mathrm{GF}_{m}$ model demonstrates skill in forecasting peak gusts: the mean $\mathrm{GF}_{m}$ model outperforms the noskill persistence ${ }_{m}$ and climatology $y_{m}$ models (Table 2). The differences in error distributions between the mean $\mathrm{GF}_{m}$ and both no-skill models are statistically significant. The seasonal/hourly peak gust climatology composing the climatology ${ }_{m}$ model is shown in the appendix.

The $\mathrm{GF}_{m}$-model performance exhibits statistically significant improvement when the gust factors used in Eq. (3) are stratified by meteorological variables, hour, and season (Table 2). Stratification by wind speed offers the largest improvement, followed by wind direction and hour/season. The best model performance occurs when the $\mathrm{GF}_{m}$ is doubly stratified by wind speed and direction. This improvement is expected, given the observed sensitivity of GFs to wind speed, surface roughness, and wind direction (Wieringa 1973; Powell et al. 2004; Harper et al. 2010; Masters et al. 2010; Pryor et al. 2014).

The GF models were also applied to a restricted evaluation period limited to hours for which gusts of $25 \mathrm{kt}$ $\left(12.9 \mathrm{~m} \mathrm{~s}^{-1}\right)$ or greater were observed (Table 3). During these gusty periods, the MAEs are larger and more variable, with mean bias ranging from $-2.1 \mathrm{kt}\left(-1 \mathrm{~m} \mathrm{~s}^{-1}\right)$ to $0.7 \mathrm{kt}\left(0.4 \mathrm{~m} \mathrm{~s}^{-1}\right)$. The principal characteristics of the $\mathrm{GF}_{m}$-model performance that were found with the unrestricted evaluation period (Table 2) were again found to be statistically significant: it is superior to the mean $\mathrm{GF}_{h}$ model; it demonstrates skill by outperforming persistence $_{m}$; its performance improves when stratified by wind speed, wind direction, and hour/season; and the best performance is achieved with a double stratification by both wind speed and wind direction. Not surprising, the climatology $_{m}$ model exhibits a smaller MAE when the observation period is restricted to gusty conditions, because the associated climatology (not shown) has the same restriction.

\section{Summary and conclusions}

A climatology of gust factors was determined for Milwaukee using ASOS wind measurements from 2007 

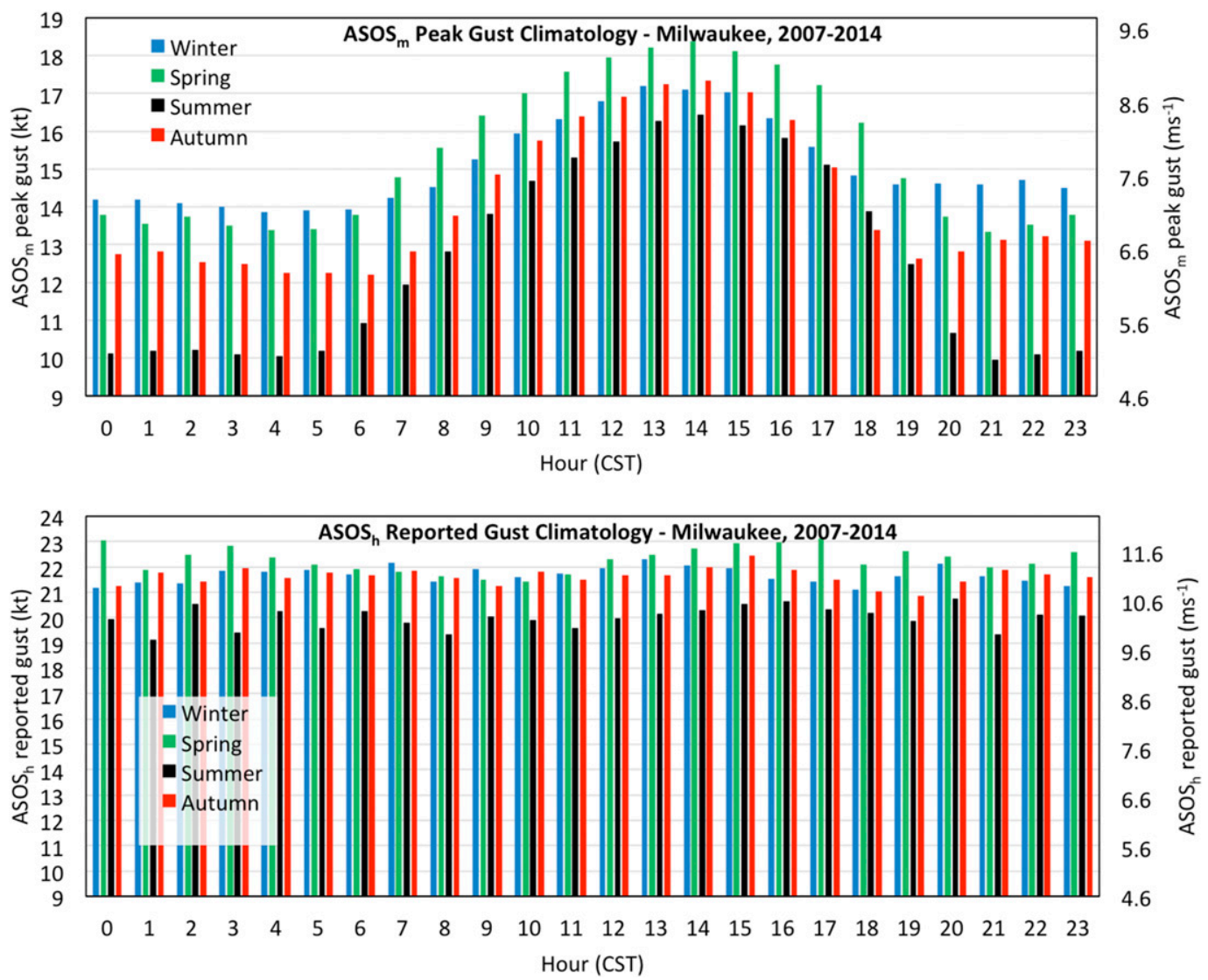

FIG. A1. Gust climatology for Milwaukee that is based on the (top) 1-min ASOS $_{m}$ and (bottom) hourly ASOS $_{h}$ datasets for 2007-14. Mean values are shown that are based on approximately $650 \mathrm{ASOS}_{m}$ peak-gust and $95 \mathrm{ASOS}_{h}$ reported gust observations during each hour and season. Standard deviations ranged from 5 to $7 \mathrm{kt}\left(2.6-3.6 \mathrm{~m} \mathrm{~s}^{-1}\right)$ for $\mathrm{ASOS}_{m}$ peak gusts and from 2 to $5 \mathrm{kt}\left(1.0-2.6 \mathrm{~m} \mathrm{~s}^{-1}\right)$ for $\mathrm{ASOS}_{h}$ reported gusts.

to 2014. Gust measurements are highly sensitive to the reporting criteria associated with the hourly $\mathrm{ASOS}_{h}$ dataset, which restrict the reporting of gusts to arbitrarily defined gusty periods occurring between minutes 43 and 52 of each hour. The reporting criteria produce the following artifacts in $\mathrm{ASOS}_{h}$ gust observations: 1) Gusts are infrequently reported in the $\mathrm{ASOS}_{h}$ hourly dataset; in Milwaukee, gusts were included in only $13 \%$ of all possible hours. 2) $\mathrm{ASOS}_{h}$ gusts are strongly biased toward higher wind speeds; gust climatologies determined using this dataset would therefore be positively skewed. The 10-min observation interval provides an additional bias in $\mathrm{ASOS}_{h}$ reported gusts, because the maximum gust during the hour normally occurs outside this interval. Even when the reporting criteria are met, therefore, the $\mathrm{ASOS}_{h}$ reported gusts underrepresent the actual peak gust. Gust factors determined from $\mathrm{ASOS}_{h}$ data are similarly affected. In Milwaukee, GFs determined using $\mathrm{ASOS}_{h}$ data are $9 \%$ smaller than those determined from the higher-resolution $\mathrm{ASOS}_{m}$ dataset and do not provide a representative measure of climatological gustiness.

Using the higher-resolution, 1-min $\operatorname{ASOS}_{m}$ dataset, a climatology of GFs was established for Milwaukee with the following principal characteristics:

1) The mean GF is 1.74.

2) GFs are sensitive to wind speed, ranging from 1.91 to 1.64 during weak and strong winds, respectively.

3) GFs are sensitive to wind direction, used in this study as a proxy for surface roughness. GFs of 1.6-1.7 were associated with winds with a long fetch over Lake Michigan, whereas values up to 1.8 occurred with inland winds.

4) GFs exhibit moderate diurnal variability, likely associated with changes in wind speed.

A variety of GF models were also evaluated for Milwaukee. In such models, the peak gust is normally estimated by multiplying a GF by a wind speed forecast. 
In this study, however, we replace the wind speed forecast with the observed wind speed during the forecast hour. When verified against observed peak gusts, the forecast errors are therefore entirely attributable to the GF model itself (i.e., to the representativeness of the GF in forecasting peak gusts). Forecast errors thus obtained are small, but model results are meaningful in a relative sense, providing insight on how to best maximize the performance of GF models.

The GF model evaluation for Milwaukee revealed the following:

1) Models using GFs derived from 1-min $\mathrm{ASOS}_{m}$ data perform better than those using hourly $\mathrm{ASOS}_{h}$ data.

2) GF models demonstrate skill in forecasting peak gusts, as compared with the no-skill models of climatology and persistence.

3) Model performance improves when GFs are stratified by wind speed, wind direction, hour, and season.

4) Double stratification by both wind speed and wind direction provides the best performance.

5) During particularly gusty conditions, the best performance occurs when GFs are doubly stratified by both wind speed and wind direction.

Our analysis suggests that GF models are a viable means of forecasting peak wind gusts. A limited analysis of similar wind and gust data for Atlanta suggests that the method may be generally applicable at other midlatitude locations. The GFs used in such models should be derived from wind and gust measurements that are free of data artifacts such as those present in the hourly $\mathrm{ASOS}_{h}$ dataset. Stratifying the GFs by controlling variables such as wind speed and direction offers a means of maximizing the performance of gustfactor models.

\section{APPENDIX}

\section{Gust Climatology for Milwaukee}

The 1-min $\operatorname{ASOS}_{m}$ peak gust climatology for Milwaukee, stratified by hour and season, is presented in Fig. A1. Early morning minima and afternoon maxima occur throughout the year, with the largest diurnal variation occurring during summer. Gusts are strongest during winter and spring and weakest during summer. Also shown is the corresponding climatology of hourly $\mathrm{ASOS}_{h}$ reported gusts, which exhibits little diurnal variability and larger, seasonally dependent mean values ranging from 19 to $23 \mathrm{kt}\left(9.8-11.8 \mathrm{~m} \mathrm{~s}^{-1}\right)$. This difference in climatologies again reveals the large biases introduced by the $\mathrm{ASOS}_{h}$ sampling periods and reporting criteria on gust measurements.

\section{REFERENCES}

Ágústsson, H., and H. Ólafsson, 2004: Mean gust factors in complex terrain. Meteor. Z., 13, 149-155, https://doi.org/10.1127/0941-2948/ 2004/0013-0149.

Beljaars, A. C. M., 1987: The measurement of gustiness at routine wind stations-A review. World Meteorological Organization Instruments and Observing Methods Rep. 31, 50 pp.

Blaes, J. L., D. Glenn, D. Hawkins, and V. Oliva, 2014: Developing a dataset of wind gust factors to improve forecasts of wind gusts in tropical cyclones. 39th National Weather Association Annual Meeting, Salt Lake City, UT, National Weather Association, P1.43.

Brasseur, O., 2001: Development and application of a physical approach to estimating wind gusts. Mon. Wea. Rev., 129, 5-25, https://doi.org/10.1175/1520-0493(2001)129<0005:

DAAOAP $>2.0 . C O ; 2$.

Carter, G., 1974: A brief study of wind gust factors. NOAA Techniques Development Laboratory Office Note 74-2, NOAA, 7 pp., http://www.nws.noaa.gov/mdl/pubs/Documents/ OfficeNotes/MDL_OfficeNote74-2.pdf.

Cheng, W. Y. Y., Y. Liu, A. J. Bourgeois, Y. Wu, and S. E. Haupt, 2017: Short-term wind forecast of a data assimilation/weather forecasting system with wind turbine anemometer measurement assimilation. Renew. Energy, 107, 340-351, https://doi.org/ 10.1016/j.renene.2017.02.014.

Choi, E. C. C., and F. A. Hidayat, 2002: Gust factors for thunderstorm and non-thunderstorm winds. J. Wind Eng. Ind. Aerodyn., 90, 1683-1696, https://doi.org/10.1016/S0167-6105(02) 00279-9.

Cook, K. R., B. Gruenbacher, and L. D. Williams, 2008: Assessment of science and methodologies to forecast wind and wind gust speed. 12th Annual High Plains Conf., Wichita, KS, National Weather Service, 21 pp.

Cook, N. J., 1986: The Designer's Guide to Wind Loading of Building Structures: Part 1: Background, Damage Survey, Wind Data and Structural Classification. Butterworth-Heinemann, 383 pp.

Davis, F. K., and H. Newstein, 1968: The variation of gust factors and mean wind speed with height. J. Appl. Meteor., 7, 372-378, https://doi.org/10.1175/1520-0450(1968)007<0372: TVOGFW $>2.0 . \mathrm{CO} ; 2$.

Friederichs, P., M. Göber, S. Bentzien, A. Lenz, and R. Krampitz, 2009: A probabilistic analysis of wind gusts using extreme value statistics. Meteor. Z., 18, 615-629, https://doi.org/ 10.1127/0941-2948/2009/0413.

Giess, P., A. J. H. Goddard, and G. Shaw, 1997: Factors affecting particle resuspension from grass swards. J. Aerosol Sci., 28 , 1331-1349, https://doi.org/10.1016/S0021-8502(97)00021-9.

Goyette, S., O. Brasseur, and M. Beniston, 2003: Application of a new wind gust parametrization: Multiscale case studies performed with the Canadian regional climate model. J. Geophys. Res., 108, 4374, https://doi.org/10.1029/2002JD002646.

Green, T. A., Jr., and R. J. Poremba, 2012: An analysis of BUFKIT methodologies to forecast wind and wind gust speed for the Upper Ohio River Valley. 37th National Weather Association Annual Meeting, Madison, WI, National Weather Association, P1.48.

Greenway, M. E., 1979: Analytical approach to wind velocity gust factors. J. Ind. Aerodyn, 5, 61-91, https://doi.org/10.1016/ 0167-6105(79)90025-4.

Harper, B. A., J. D. Kepert, and J. D. Ginger, 2010: Guidelines for converting between various wind averaging periods in tropical cyclone conditions. World Meteorological Organization Tech. 
Doc. WMO/TD-1555, 64 pp., https://www.wmo.int/pages/ prog/www/tcp/documents/WMO_TD_1555_en.pdf.

Hart, R. E., and G. S. Forbes, 1999: The use of hourly modelgenerated soundings to forecast mesoscale phenomena. Part II: Initial assessment in forecasting nonconvective strong wind gusts. Wea. Forecasting, 14, 461-469, https://doi.org/10.1175/ 1520-0434(1999)014<0461:TUOHMG > 2.0.CO;2.

Houghton, D. D., 1985: Handbook of Applied Meteorology. WileyInterscience, $1461 \mathrm{pp}$.

Huschke, R. E., Ed., 1959: Glossary of Meteorology. Amer. Meteor. Soc., $638 \mathrm{pp}$.

Jensen, N. O., 1999: Atmospheric boundary layers and turbulence. Proc. 10th Int. Conf. on Wind Engineering, Copenhagen, Denmark, International Association of Wind Engineering, 29-42.

Johnson, D. L., and R. J. Erhardt, 2016: Projected impacts of climate change on wind energy density in the United States. Renew. Energy, 85, 66-73, https://doi.org/10.1016/ j.renene.2015.06.005.

Jung, C., and D. Schindler, 2016: Modelling monthly near-surface maximum daily gust speed distributions in southwest Germany Int. J. Climatol., 36, 4058-4070, https://doi.org/10.1002/joc.4617.

,,-- A. T. Albrecht, and A. Bucholz, 2016: The role of highlyresolved gust speed in simulations of storm damage in forests at the landscape scale: A case study from southwest Germany. Atmosphere, 7, 7, https://doi.org/10.3390/atmos7010007.

Kramer, R., and F. Alsheimer, 2013: Wind gust climatology for southern South Carolina and coastal north Georgia. National Weather Service Presentation, 31 pp., www.weather.gov/ media/chs/research/Kramer-WindGustClimo.pdf.

Kristensen, L., M. Casanova, M. S. Courtney, and I. Troen, 1991: In search of a gust definition. Bound.-Layer Meteor., 55, 91-107, https://doi.org/10.1007/BF00119328.

Larsén, X. G., S. Ott, J. Badger, A. N. Hahmann, and J. Mann, 2012: Recipes for correcting the impact of effective mesoscale resolution on the estimation of extreme winds. J. Appl. Meteor. Climatol., 51, 521-533, https://doi.org/10.1175/ JAMC-D-11-090.1.

Lombardo, F. T., J. A. Main, and E. Simiu, 2009: Automated extraction and classification of thunderstorm and nonthunderstorm wind data for extreme-value analysis. J. Wind Eng. Ind. Aerodyn., 97, 120-131, https://doi.org/ 10.1016/j.jweia.2009.03.001.

Luna, R. E., and H. W. Church, 1972: A comparison of turbulence intensity and stability ratio measurements to Pasquill stability classes. J. Appl. Meteor., 11, 663-669, https://doi.org/10.1175/ 1520-0450(1972)011<0663:ACOTIA > 2.0.CO;2.

Manasseh, R., and J. H. Middleton, 1999: The surface wind gust regime and aircraft operations at Sydney Airport. J. Wind Eng. Ind. Aerodyn., 79, 269-288, https://doi.org/10.1016/ S0167-6105(97)00293-6.

Masters, F. J., P. J. Vickery, P. Bacon, and E. N. Rappaport, 2010: Toward objective, standardized intensity estimates from surface wind speed observations. Bull. Amer. Meteor. Soc., 91, 1665-1682, https://doi.org/10.1175/2010BAMS2942.1.

Mendenhall, W., D. D. Wackerly, and R. L. Schaeffer, 1990: Mathematical Statistics with Applications. 4th ed. PWS-Kent Publishing, $818 \mathrm{pp}$.

Mitsuta, Y., and O. Tsukamoto, 1989: Studies on spatial structure of wind gust. J. Appl. Meteor., 28, 1155-1160, https://doi.org/ 10.1175/1520-0450(1989)028<1155:SOSSOW $>2.0$.CO;2.

NOAA, 1998: Automated Surface Observing System (ASOS) user's guide. NOAA Tech. Doc., 72 pp., http://www.nws.noaa.gov/ asos/aum-toc.pdf.
Orwig, K. D., and J. L. Schroeder, 2007: Near-surface wind characteristics of extreme thunderstorm outflows. J. Wind Eng. Ind. Aerodyn., 95, 565-584, https://doi.org/10.1016/j.jweia.2006.12.002.

Panofsky, H. A., and J. A. Dutton, 1984: Atmospheric Turbulence: Models and Methods for Engineering Applications. John Wiley and Sons, $389 \mathrm{pp}$.

Pasquill, F., 1961: The estimation of the dispersion of windborne material. Meteor. Mag., 90, 33-49.

Paulsen, B. M., and J. L. Schroeder, 2005: An examination of tropical and extratropical gust factors and the associated wind speed histograms. J. Appl. Meteor., 44, 270-280, https://doi.org/ 10.1175/JAM2199.1

Powell, M. D., S. Houston, and T. A. Reinhold, 1996: Hurricane Andrew's landfall in south Florida. Part I: Standardizing measurements for documentation of surface wind fields. Wea. Forecasting, 11, 304-328, https://doi.org/10.1175/1520-0434(1996)011<0304: HALISF $>2.0 . \mathrm{CO} ; 2$.

, D. Bowman, D. Gilhousen, S. Murillo, N. Carrasco, and R.St. Fleur, 2004: Tropical cyclone winds at landfall: The ASOS-CMAN wind exposure documentation project. Bull. Amer. Meteor. Soc., 85, 845-851, https://doi.org/10.1175/BAMS-85-6-845.

Pryor, S. C., R. Conrick, C. Miller, J. Tytell, and R. J. Barthelmie, 2014: Intense and extreme wind speeds observed by anemometer and seismic networks: An eastern U.S. case study. J. Appl. Meteor. Climatol., 53, 2417-2429, https://doi.org/ 10.1175/JAMC-D-14-0091.1.

Rudack, D., 2006: GFS-based MOS wind gust guidance for the United States, Puerto Rico, and the U.S. Virgin Islands. NOAA MDL Tech. Procedures Bull. 06-01, 9 pp., http://www.nws.noaa.gov/ $\mathrm{mdl} / \mathrm{synop} / \mathrm{tpb} / \mathrm{mdltpb} 06-01 . p d f$.

Schulz, J. P., 2008: Revision of the turbulent gust diagnostics in the COSMO model. COSMO Newsletter, No. 8, Consortium for SmallScale Modeling, 17-22, http://www.cosmo-model.org/content/ model/documentation/newsLetters/newsLetter08/default.htm.

Shellard, H. C., 1965: The estimation of design wind speeds. Proc. 16th Symp. on Wind Effects on Building and Structures, Teddington, Middlesex, United Kingdom, National Physical Laboratory, 30-51.

Sherlock, R. H., 1952: Variation of wind velocity and gust with height. Proc. Amer. Soc. Civ. Eng., Vol. 78 (Separate No. 126; Paper 2553), $26 \mathrm{pp}$.

Soe, T. T., and A. A. Thant, 2014: Comparative study on wind averaging conversion between Engineering Sciences Data Unit (ESDU) gust factor method and statistical approach. Int. J. Sci. Eng. Tech. Res., 3, 1681-1687.

Suomi, I., T. Vihma, S.-E. Gryning, and C. Fortelius, 2013: Windgust parametrizations at heights relevant for wind energy: A study based on mast observations. Quart. J. Roy. Meteor. Soc., 139, 1298-1310, https://doi.org/10.1002/qj.2039.

— S.-E. Gryning, R. Floors, T. Vihma, and C. Fortelius, 2015: On the vertical structure of wind gusts. Quart. J. Roy. Meteor. Soc., 141, 1658-1670, https://doi.org/10.1002/qj.2468.

C. Lupkes, J. Hartmann, T. Vihma, S.-E. Gryning, and C. Fortelius, 2016: Gust factor based on research aircraft measurements: A new methodology applied to the Arctic marine boundary layer. Quart. J. Roy. Meteor. Soc., 142, 29853000, https://doi.org/10.1002/qj.2880.

Traiteur, J. J., D. J. Callicutt, M. Smith, and S. B. Roy, 2012: A short-term ensemble wind speed forecasting system for wind power applications. J. Appl. Meteor. Climatol, 51, 1763-1774, https://doi.org/10.1175/JAMC-D-11-0122.1.

Van der Hoven, I., 1957: Power spectrum of horizontal wind speed in the frequency range from 0.0007 to 900 cycles per hour. 
J. Meteor., 14, 160-164, https://doi.org/10.1175/1520-0469 (1957)014<0160:PSOHWS>2.0.CO;2.

Wichers Schreur, B., and G. Geertsema, 2008: Theory for a TKE based parameterization of wind gusts. HIRLAM Newsletter, No. 54, High Resolution Limited Area Model Consortium, 177-188.

Wieringa, J., 1973: Gust factors over open water and built-up country. Bound.-Layer Meteor., 3, 424-441, https://doi.org/ 10.1007/BF01034986.

Woetmann Nielsen, N., and C. Petersen, 2001: Calculation of wind gusts in DMI-HIRLAM. Danish Meteorological Institute
Scientific Rep. 01-03, 33 pp, https://www.dmi.dk/fileadmin/ Rapporter/SR/sr01-03.pdf.

Wong, C. J., and M. D. Miller, Eds., 2010: Guidelines for Electrical Transmission Line Structural Loading. 3rd ed., ASCE Manuals and Reports on Engineering Practice, Vol. 74, Amer. Soc. Civil Engineers, 204 pp.

Zamo, M., L. Bel, O. Mestre, and J. Stein, 2016: Improved gridded wind speed forecasts by statistical postprocessing of numerical models with block regression. Wea. Forecasting, 31, 19291945, https://doi.org/10.1175/WAF-D-16-0052.1. 